Bull. Austral. Math. Soc.

Vol. 53 (1996) [1-12]

\title{
AN ANALOGUE OF PROBLEM 26 OF P. TURÁN
}

\author{
Y.G. SHI
}

Explicit formulas for Cotes numbers of the Gaussian Hermite quadrature formula based on the zeros of the $n$th Chebyshev polynomial of the second kind and their asymptotic behaviour as $n \rightarrow \infty$ are given. This provides an answer to an analogue of Problem 26 of Turán.

\section{INTRODUCTION AND MAIN RESULTS}

This paper deals with explicit formulas for Cotes numbers of the Gaussian Hermite quadrature formula based on the zeros of the $n$th Chebyshev polynomial of the second kind and their asymptotic behaviour as $n \rightarrow \infty$.

Let $\alpha(x)$ be a nonnegative function on $[-1,1]$ with infinitely many points of increase such that all moments of $\alpha(x)$ are finite and let $\mathbf{P}_{N}$ denote the set of polynomials of degree $\leqslant N$. According to Theorem 4 in [3], given integers $m \geqslant 1$ and $p \geqslant 0$, if $\omega_{n}(x):=\prod_{k=1}^{n}\left(x-x_{k n}\right)$ with

$$
1=x_{0 n}>x_{1 n}>x_{2 n}>\cdots>x_{n n}>x_{n+1, n}=-1, \quad n \geqslant 1
$$

satisfies

$$
\int_{-1}^{1}\left(1-x^{2}\right)^{p}\left|\omega_{n}(x)\right|^{m} d \alpha(x)=\min _{P=x^{n}+\ldots} \int_{-1}^{1}\left(1-x^{2}\right)^{p}|P(x)|^{m} d \alpha(x),
$$

then the quadrature formula with certain numbers $c_{i k m}:=c_{i k m n}$ (called Cotes numbers of higher order)

$$
\int_{-1}^{1} f(x) \sigma(x) d \alpha(x)=\sum_{k=0}^{n+1} \sum_{i=0}^{\mu_{k}} c_{i k m} f^{(i)}\left(x_{k}\right)
$$

is exact for all $f \in \mathbf{P}_{m n+2 p-1}$, where

$$
\sigma(x):=\operatorname{sgn} \omega_{n}(x)^{m}
$$

Received 24 February 1995

The project was supported by the National Natural Science Foundation of China.

Copyright Clearance Centre, Inc. Serial-fee code: 0004-9729/96 \$A2.00+0.00. 
and

$$
\mu_{k}:= \begin{cases}m-2, & 1 \leqslant k \leqslant n \\ p-1, & \text { otherwise }\end{cases}
$$

As Turán pointed out in $[10$, p.46], particularly interesting is the case

$$
p=0, \quad d \alpha(x)=\frac{d x}{\sqrt{1-x^{2}}}
$$

By a theorem of Bernstein [2], in this case the $n$th Chebyshev polynomial of first kind $2^{1-n} T_{n}(x)$ is the solution of (1.2) for all values of $m \geqslant 1$. Meanwile, Turán raised the following problem for even $m\left[10\right.$, p.47], in which $c_{i k m n}$ stands for the Cotes number based on the zeros of $T_{n}(x)$ :

PROBLEM 26. Give an explicit formula for $c_{i k m n}$ and determine its asymptotic behaviour as $n \rightarrow \infty$.

In $[6,7]$ we got a solution of this problem for each (even and odd) $m$.

Another particular interesting case is

$$
p=\left[\frac{m}{2}\right], d \alpha(x)=d \alpha_{m}(x):=\left(1-x^{2}\right)^{[(m+1) / 2]-(m+1) / 2} d x
$$

By a well known result (see, say, [8]), in this case the $n$th Chebyshev polynomial of the second kind $2^{-n} U_{n}(x)$ is the solution of (1.2) for all values of $m \geqslant 1$. Then the quadrature formula with certain numbers $c_{i k m}$

$$
\int_{-1}^{1} f(x) \sigma_{m}(x) d \alpha_{m}(x)=\sum_{k=0}^{n+1} \sum_{i=0}^{m_{k}} c_{i k m} f^{(i)}\left(x_{k}\right)
$$

is exact for all $f \in \mathbf{P}_{m n+2[m / 2]-1}$, where

$$
\sigma_{m}(x):=\operatorname{sgn} U_{n}(x)^{m}
$$

and

$$
m_{k}:=\left[n_{k}(m-2)\right], \quad n_{k}:= \begin{cases}1, & 1 \leqslant k \leqslant n \\ \frac{1}{2}, & \text { otherwise }\end{cases}
$$

In the present paper, using a modification of the main idea of [6], we intend to answer to the same problem for this quadrature formula. It turns out that, in the 
present case the problem is more difficult and complicated. To state our results we need the notation:

$$
\begin{aligned}
& l_{k}(x):=l_{k n}(x):=\frac{\omega_{n}(x)}{\left(x-x_{k}\right) \omega_{n}^{\prime}\left(x_{k}\right)}, \quad k=1,2, \ldots, n, \\
& \Delta_{m}(x):=\Delta_{m n}(x):=\left(1-x^{2}\right)^{[m / 2]} U_{n}(x)^{m} \text {, } \\
& d_{k m}:=\Delta_{m n}^{\left(\left[n_{k} m\right]\right)}\left(x_{k}\right)= \begin{cases}m !\left(1-x_{k}^{2}\right)^{[m / 2]} U_{n}^{\prime}\left(x_{k}\right)^{m}, & 1 \leqslant k \leqslant n, \\
(-2)^{[m / 2]}\left(\left[\frac{m}{2}\right]\right) ! U_{n}(1)^{m}, & k=0, \\
2^{[m / 2]}\left(\left[\frac{m}{2}\right]\right) ! U_{n}(-1)^{m}, & k=n+1,\end{cases} \\
& L_{k m n}(x):=L_{k m n}(x):=\frac{\left(\left[n_{k} m\right]\right) ! \Delta_{m n}(x)}{d_{k m}\left(x-x_{k}\right)^{\left[n_{k} m\right]}}, \quad k=0,1, \ldots, n+1 \text {, } \\
& b_{i k m}:=b_{i k m n}:=\frac{1}{i !}\left[L_{k m}(x)^{-1}\right]_{x=x_{k}}^{(i)}, \quad i=0,1, \ldots ; k=0,1, \ldots, n+1 \text {, } \\
& B_{i k m}:=B_{i k m n}:=\frac{1}{i !}\left\{\sum_{\nu \in\{0, n+1\} \backslash\{k\}}\left[2\left(x_{\nu}-x\right) L_{k m}(x)\right]^{-1}\right\}_{x=x_{k}}^{(i)}, \\
& i=0,1, \ldots ; k=0,1, \ldots, n+1,
\end{aligned}
$$

The main results in this paper is

ThEOREM. Let (1.1) be the zeros of $\Omega_{n}(x):=\left(1-x^{2}\right) U_{n}(x)$ and let $m \geqslant 1$ be an integer. Then for each $i, 0 \leqslant i \leqslant m_{k}$, and for each $k, 0 \leqslant k \leqslant n+1$,

$$
\left\{\begin{array}{l}
c_{m_{k}, k, m}=\frac{n_{k} s_{m}(m-2) !}{d_{k, m-2}[(m-2) ! !]^{2}(n+1)}, \quad m \geqslant 2, \\
c_{m_{k}+1, k, m}=0,
\end{array}\right.
$$

$$
\begin{aligned}
c_{i k m}=c_{i, k, m-2}+\frac{m_{k} ! c_{m_{k}, k, m}}{i ! n_{k}(m-2)} & \left\{\left(i+n_{k}(m-2)-m_{k}\right) b_{m_{k}-i, k, m-2}\right. \\
& \left.-\frac{1}{2}\left[1+(-1)^{m+1}\right] B_{m_{k}-i-1, k, m-2}\right\}, \quad m \geqslant 3 .
\end{aligned}
$$

Moreover

$$
\left|c_{i k m n}\right| \leqslant \begin{cases}\frac{\left(1-x_{k n}^{2}\right)^{[(m-1) / 2]-[(m-1-i) / 2]}}{n^{m+1-2 \mid(m-i) / 2]}}, & 1 \leqslant k \leqslant n \\ \frac{1}{n^{m-2[m / 2]+2 i+1}}, & \text { otherwise }\end{cases}
$$

(1.19) $c_{i k m n} \sim \frac{\left(1-x_{k n}^{2}\right)^{i / 2}}{n^{i+1}}, m=$ even; $i=0,2,4, \ldots, m-2 ; 1 \leqslant k \leqslant n$. 
REMARK. For sake of notation we still use the idex $m$ in (1.16) and (1.17). In fact, they remain true if we replace $m$ by any suitable integer $r$ (say, $r \geqslant 3$ in (1.17)). Thus these two formulas provide an explicit expression for $c_{i k m}$.

\section{LEMMAS}

To prove our theorem we need several lemmas.

LEMMA 1. Let

$$
a_{i k}:=a_{i k m n}:=\sum_{\substack{\nu=0 \\ \nu \neq k}}^{n+1} \frac{\left[n_{\nu} m\right]}{\left(x_{\nu}-x_{k}\right)^{i}}, i=1,2, \ldots ; 0 \leqslant k \leqslant n+1 .
$$

Then

$$
b_{i k m n}=\frac{1}{i} \sum_{\nu=1}^{i} a_{\nu k} b_{i-\nu, k, m}, i=1,2, \ldots ; 0 \leqslant k \leqslant n+1 .
$$

Proof: Let $k, 0 \leqslant k \leqslant n+1$, be fixed. It is easy to check that

$$
\frac{L_{k m}^{\prime}(x)}{L_{k m}(x)}=-\sum_{\substack{\nu=0 \\ \nu \neq k}}^{n+1} \frac{\left[n_{\nu} m\right]}{x \nu-x}
$$

Hence

$$
a_{i k}=\frac{1}{(i-1) !}\left[-\frac{L_{k m}^{\prime}(x)}{L_{k m}(x)}\right]_{x=x_{k}}^{(i-1)}
$$

Using (1.13) and applying the Newton-Leibniz rule we get

$$
\begin{aligned}
\frac{1}{i} \sum_{\nu=1}^{i} a_{\nu k} b_{i-\nu, k, m} & =\frac{1}{i} \sum_{\nu=1}^{i} \frac{1}{(\nu-1) !}\left[-\frac{L_{k m}^{\prime}(x)}{L_{k m}(x)}\right]_{x=x_{k}}^{(\nu-1)} \frac{1}{(i-\nu) !}\left[L_{k m}(x)^{-1}\right]_{x=x_{k}}^{(i-\nu)} \\
& =\frac{1}{i !}\left[-\frac{L_{k m}^{\prime}(x)}{L_{k m}(x)} L_{k m}(x)^{-1}\right]_{x=x_{k}}^{(i-1)}=\frac{1}{i !}\left[L_{k m}(x)^{-1}\right]_{x=x_{k}}^{(i)}=b_{i k m} .
\end{aligned}
$$

Lemma 2. Let (1.1) be the zeros of $\Omega_{n}(x)$ and let $m \geqslant 0$ be an integer. Then for each $k, 0 \leqslant k \leqslant n+1$,

$$
\int_{-1}^{1} \frac{\left|\left(1-x^{2}\right)^{1 / 2} U_{n}(x)\right|^{m} \Omega_{n}(x)}{\Omega_{n}^{\prime}\left(x_{k}\right)\left(x-x_{k}\right)} \frac{d x}{\sqrt{1-x^{2}}}=\frac{n_{k} s_{m}(m !)}{(m ! !)^{2}(n+1)} .
$$

ProOF: We distinguish two cases. 
CASE $1.1 \leqslant k \leqslant n$.

We know [5, Volume 3, Chapter 5, Section 4]

$$
\int_{-1}^{1} l_{k}(x)\left(1-x^{2}\right)^{1 / 2} d x=\frac{\pi\left(1-x_{k}^{2}\right)}{n+1}
$$

Expand $|\sin \theta|^{m}$ as

$$
|\sin \theta|^{m}=\sum_{\nu=0}^{\infty} \beta_{\nu} \cos \nu \theta
$$

Here we can use [4, Formula 3.621-3 and 4, p.369] to calculate the required first two coefficients:

$$
\beta_{0}=\frac{1}{\pi} \int_{0}^{\pi}|\sin \theta|^{m} d \theta=\frac{s_{m}(m !)}{\pi(m ! !)^{2}}, \quad \beta_{1}=0 .
$$

Replacing $\theta$ by $(n+1) \theta$ in $(2.7)$ yields

$$
|\sin (n+1) \theta|^{m}=\sum_{\nu=0}^{\infty} \beta_{\nu} \cos \nu(n+1) \theta
$$

By making the substitution $\theta=\arccos x$, we obtain

$$
\left|\left(1-x^{2}\right)^{1 / 2} U_{n}(x)\right|^{m}=\sum_{\nu=0}^{\infty} \beta_{\nu} T_{\nu(n+1)}(x) .
$$

Then

$$
\begin{aligned}
\int_{-1}^{1} \frac{\left|\left(1-x^{2}\right)^{1 / 2} U_{n}(x)\right|^{m} \Omega_{n}(x)}{\Omega_{n}^{\prime}\left(x_{k}\right)\left(x-x_{k}\right)} \frac{d x}{\sqrt{1-x^{2}}} \\
\quad=\frac{1}{1-x_{k}^{2}} \int_{-1}^{1}\left|\left(1-x^{2}\right)^{1 / 2} U_{n}(x)\right|^{m} l_{k}(x)\left(1-x^{2}\right) \frac{d x}{\sqrt{1-x^{2}}} \\
=\frac{1}{1-x_{k}^{2}} \int_{-1}^{1}\left[\sum_{\nu=0}^{\infty} \beta_{\nu} T_{\nu(n+1)}(x)\right] l_{k}(x)\left(1-x^{2}\right) \frac{d x}{\sqrt{1-x^{2}}} \\
=\frac{\beta_{0}}{1-x_{k}^{2}} \int_{-1}^{1} l_{k}(x)\left(1-x^{2}\right)^{1 / 2} d x=\frac{s_{m}(m !)}{(m ! !)^{2}(n+1)}
\end{aligned}
$$

CAse 2. $k=0, n+1$. 
We give the proof for the case $k=0$ only, the proof for the case $k=n+1$ being similar. Since [1, Formula 22.3.12, p.776]

$$
U_{n}(\cos \theta)=\frac{\sin (n+1) \theta}{\sin \theta}=\sum_{\nu=0}^{n} \cos (n-2 \nu) \theta
$$

we have

$$
\int_{0}^{\pi} U_{n}(\cos \theta)(1+\cos \theta) d \theta=\pi
$$

This means

$$
\int_{-1}^{1} U_{n}(x)(1+x) \frac{d x}{\sqrt{1-x^{2}}}=\pi
$$

By (2.8) and (2.10) we conclude

$$
\begin{aligned}
\int_{-1}^{1} \frac{\left|\left(1-x^{2}\right)^{1 / 2} U_{n}(x)\right|^{m} \Omega_{n}(x)}{\Omega_{n}^{\prime}(1)(x-1)} \frac{d x}{\sqrt{1-x^{2}}} \\
\quad=\frac{1}{2(n+1)} \int_{-1}^{1}\left|\left(1-x^{2}\right)^{1 / 2} U_{n}(x)\right|^{m} U_{n}(x)(1+x) \frac{d x}{\sqrt{1-x^{2}}} \\
=\frac{1}{2(n+1)} \int_{-1}^{1}\left[\sum_{\nu=0}^{\infty} \beta_{\nu} T_{\nu(n+1)}(x)\right] U_{n}(x)(1+x) \frac{d x}{\sqrt{1-x^{2}}} \\
=\frac{\beta_{0}}{2(n-1)} \int_{-1}^{1} U_{n}(x)(1+x) \frac{d x}{\sqrt{1-x^{2}}}=\frac{s_{m}(m !)}{2(m ! !)^{2}(n+1)}
\end{aligned}
$$

LEMMA 3. Let (1.1) be the zeros of $\Omega_{n}(x)$ and let $m \geqslant 1$ be an integer. Then for each $i, i<\left[n_{k} m\right]$,

$$
\begin{aligned}
& \left|b_{i k m n}\right| \leqslant \begin{cases}c \frac{n^{2[i / 2]}}{\left(1-x_{k n}^{2}\right)^{[(i+1) / 2]}}, & 1 \leqslant k \leqslant n \\
c n^{2 i}, & \text { otherwise, }\end{cases} \\
& \left|B_{i k m n}\right| \leqslant \begin{cases}c \frac{n^{2[i / 2]}}{\left(1-x_{k n}^{2}\right)^{[(i+3) / 2]},} & 1 \leqslant k \leqslant n \\
c n^{2 i}, & \text { otherwise. }\end{cases}
\end{aligned}
$$

Proof: We distinguish two cases. 
CASE $1.1 \leqslant k \leqslant n$.

We have [11, Lemma 3.11]

$$
\left|\left[l_{k}(x)^{-m}\right]_{x=x_{k}}^{(j)}\right| \leqslant c_{1} \frac{n^{2[j / 2]}}{\left(1-x_{k}^{2}\right)^{[(j+1) / 2]}}, \quad j<m
$$

Meanwhile it is easy to see that

$$
\left|\left[\left(\frac{1-x^{2}}{1-x_{k}^{2}}\right)^{-[m / 2]}\right]_{x=x_{k}}^{(j)}\right| \leqslant c_{2}\left(1-x_{k}^{2}\right)^{-j}, j<m .
$$

Then by the Newton-Leibniz rule

$$
\left|b_{i k m}\right|=\left|\frac{1}{i !}\left[\left(\frac{1-x^{2}}{1-x_{k}^{2}}\right)^{-[m / 2]} l_{k}(x)^{-m}\right]_{x=x_{k}}^{(i)}\right| \leqslant c \frac{n^{2[i / 2]}}{\left(1-x_{k}^{2}\right)^{[(i+1) / 2]}} .
$$

Here we used the relation

$$
\left(1-x_{k}^{2}\right)^{-1 / 2} \leqslant c_{3} n
$$

On the other hand, since in this case

$$
B_{i k m}=\frac{1}{2 i !}\left[\left(\frac{1}{1-x}-\frac{1}{1+x}\right) L_{k m}(x)^{-1}\right]_{x=x_{k}}^{(i)},
$$

we have in a similar way

$$
\left|B_{i k m}\right| \leqslant c \frac{n^{2[i / 2]}}{\left(1-x_{k}^{2}\right)^{[(i+3) / 2]}}
$$

CASE 2. $k=0, n+1$.

We discuss the case $k=0$, the case $k=n+1$ leading to entirely analogous, symmetric considerations. In this case we obtain

$$
\left|b_{i, 0, m}\right|=\left|\frac{1}{i !}\left[\left(\frac{1+x}{2}\right)^{-[m / 2]}\left(\frac{U_{n}(x)}{U_{n}(1)}\right)^{-m}\right]_{x=1}^{(i)}\right| \leqslant c n^{2 i} .
$$

Meanwhile

$$
\left|B_{i, 0, m}\right|=\left|\frac{1}{2 i !}\left[(1+x)^{-1} L_{0, m}(x)^{-1}\right]_{x=1}^{(i)}\right| \leqslant c n^{2 i} .
$$


LEMмA 4. Let (1.1) be the zeros of $\Omega_{n}(x)$ and let $m \geqslant 1$ be an integer. Then (1.16) is true for each $k, 0 \leqslant l \leqslant n+1$.

Proof: That $c_{0, k, 1}=0,0 \leqslant k \leqslant n+1$, follows from (1.7). Now let $m \geqslant 2$ and let $k, 0 \leqslant k \leqslant n+1$, be fixed. It is easy to see that

$$
f(x)=\frac{\Delta_{m-2}(x) \Omega_{n}(x)}{d_{k, m-2} \Omega_{n}^{\prime}\left(x_{k}\right)\left(x-x_{k}\right)}
$$

satisfies the conditions:

$$
f^{(i)}\left(x_{j}\right)=\delta_{i, m_{k}} \delta_{j k}, i=0,1, \ldots, m_{k} ; j=0,1, \ldots, n+1 .
$$

In fact, it is sufficient to show

$$
f^{\left(m_{k}\right)}\left(x_{k}\right)=1
$$

By the Newton-Leibniz rule and (1.11) this is indeed the case:

$$
f^{\left(m_{k}\right)}\left(x_{k}\right)=\left[\frac{\Delta_{m-2}(x) \Omega_{n}(x)}{d_{k, m-2} \Omega_{n}^{\prime}\left(x_{k}\right)\left(x-x_{k}\right)}\right]_{x=x_{k}}^{\left(m_{k}\right)}=\frac{1}{d_{k, m-2}} \Delta_{m-2}^{\left(m_{k}\right)}\left(x_{k}\right)=1 .
$$

Substituting $f$ into (1.7) and using (2.5) gives

$$
\begin{aligned}
c_{m_{k}, k, m} & =\int_{-1}^{1} f(x) \sigma_{m}(x) d \alpha_{m}(x) \\
& =\frac{1}{d_{k, m-2}} \int_{-1}^{1} \frac{\left|\left(1-x^{2}\right)^{1 / 2} U_{n}(x)\right|^{m-2} \Omega_{n}(x)}{\Omega_{n}^{\prime}\left(x_{k}\right)\left(x-x_{k}\right)} \frac{d x}{\sqrt{1-x^{2}}} \\
& =\frac{n_{k} s_{m}(m-2) !}{d_{k, m-2}[(m-2) ! !]^{2}(n+1)}
\end{aligned}
$$

\section{Proof of THEOREM}

The theorem for $m \leqslant 2$ or $i=m_{k}$ is given by Lemma 4 . Now assume that $m \geqslant 3$ and that both $i$ and $k, 0 \leqslant i \leqslant m_{k}-1,0 \leqslant k \leqslant n+1$, are fixed.

By the same arguments as that in [9, Lemma 1] we conclude

$$
f(x)=\frac{1}{i !} \sum_{j=0}^{m_{k}-1-i} b_{j, k, m-2}\left(x-x_{k}\right)^{i+j} L_{k, m-2}(x)
$$

satisfies the conditions:

$$
f^{\mu}\left(x_{\nu}\right)=\delta_{i \mu} \delta_{k \nu}, \mu=0,1, \ldots, m_{k}-1 ; \nu=0,1, \ldots, n+1
$$


[9]

An analogue of problem 26 of P. Turán

9

Applying (1.7) to $f$ twice yields

$$
\begin{aligned}
c_{i, k, m-2} & =\int_{-1}^{1} f(x) \sigma_{m-2}(x) d \alpha_{m-2}(x) \\
& =\int_{-1}^{1} f(x) \sigma_{m}(x) d \alpha_{m}(x)=c_{i k m}+\sum_{\nu=0}^{n+1} c_{m_{\nu}, \nu, m} f^{\left(m_{\nu}\right)}\left(x_{\nu}\right) .
\end{aligned}
$$

We distinguish two cases.

CASE 1. $\nu=k$.

From (1.11) and (1.12) it follows that $L_{k m}\left(x_{k}\right)=1$. Using (3.1) and (2.5) and applying the Newton-Leibniz rule twice, we obtain

$$
\begin{aligned}
f^{\left(m_{k}\right)}\left(x_{k}\right) & =\frac{1}{i !} \sum_{j=0}^{m_{k}-1-i} b_{j, k, m-2}\left[\left(x-x_{k}\right)^{i+j} L_{k, m-2}(x)\right]_{x=x_{k}}^{\left(m_{k}\right)} \\
& =\frac{\left(m_{k}\right) !}{i !} \sum_{j=0}^{m_{k}-1-i} b_{j, k, m-2} \frac{L_{k, m-2}^{\left(m_{k}-i-j\right)}\left(x_{k}\right)}{\left(m_{k}-i-j\right) !} \\
& =\left(\begin{array}{c}
m_{k} \\
i
\end{array}\right) \sum_{j=0}^{m_{k}-1-i}\left(\begin{array}{c}
m_{k}-i \\
j
\end{array}\right)\left[L_{k, m-2}(x)^{-1}\right]_{x=x_{k}}^{(j)} \frac{L_{k, m-2}^{\left(m_{k}-i-j\right)}\left(x_{k}\right)}{\left(m_{k}-i-j\right) !} \\
& =\left(\begin{array}{c}
m_{k} \\
i
\end{array}\right)\left\{[1]_{x=x_{k}}^{\left(m_{k}-i\right)}-\left(m_{k}-i\right) ! b_{m_{k}-i, k, m-2}\right\} \\
& =-\frac{\left(m_{k}\right) ! b_{m_{k}-i, k, m-2}}{i !} .
\end{aligned}
$$

CASE 2. $\nu \neq k$.

By using (3.1) and applying the Newton-Leibniz rule again we have

$$
\begin{aligned}
\sum_{\nu \neq k} c_{m_{\nu}, \nu, m} f^{\left(m_{\nu}\right)}\left(x_{\nu}\right) & =\frac{1}{i !} \sum_{\nu \neq k} c_{m_{\nu}, \nu, m} \sum_{j=0}^{m_{k}-1-i} b_{j, k, m-2}\left[\left(x-x_{k}\right)^{i+j} L_{k, m-2}(x)\right]_{x=x_{\nu}}^{\left(m_{\nu}\right)} \\
& =\frac{1}{i !} \sum_{j=0}^{m_{k}-1-i} b_{j, k, m-2} \sum_{\nu \neq k} c_{m_{\nu}, \nu, m}\left[\left(x-x_{k}\right)^{i+j} L_{k, m-2}(x)\right]_{x=x_{\nu}}^{\left(m_{\nu}\right)} \\
& =\frac{1}{i !} \sum_{j=0}^{m_{k}-1-i} b_{j, k, m-2} \sum_{\nu \neq k} c_{m_{\nu}, \nu, m}\left(x_{\nu}-x_{k}\right)^{i+j} L_{k, m-2}^{\left(m_{\nu}\right)}\left(x_{\nu}\right) .
\end{aligned}
$$

According to (1.11) and (1.12) we see that

$$
L_{k, m-2}^{\left(m_{\nu}\right)}\left(x_{\nu}\right)=\frac{d_{\nu, m-2} m_{k} !}{d_{k, m-2}\left(x_{\nu}-x_{k}\right)^{m_{k}}}, \quad \nu \neq k
$$

https://doi.org/10.1017/S000497270001666X Published online by Cambridge University Press 
Substituting (1.16) and (3.4) into the previous formula and applying Lemma 1, we get

$$
\begin{aligned}
& \sum_{\nu \neq k} c_{m_{\nu}, \nu, m} f^{\left(m_{\nu}\right)}\left(x_{\nu}\right) \\
& =\frac{s_{m}(m-2) ! m_{k} !}{i ![(m-2) ! !]^{2}(n+1) d_{k, m-2}} \sum_{j=0}^{m_{k}-1-i} b_{j, k, m-2} \sum_{\nu \neq k} \frac{n_{\nu}}{\left(x_{\nu}-x_{k}\right)^{m_{k}-i-j}} \\
& =\frac{m_{k} ! c_{m_{k}, k, m}}{i ! n_{k}(m-2)} \sum_{j=0}^{m_{k}-1-i} b_{j, k, m-2} \sum_{\nu \neq k} \frac{n_{\nu}(m-2)}{\left(x_{\nu}-x_{k}\right)^{m_{k}-i-j}} \\
& =\frac{m_{k} ! c_{m_{k}, k, m}}{i ! n_{k}(m-2)} \sum_{j=0}^{m_{k}-1-i} b_{j, k, m-2} \sum_{\nu \neq k} \frac{m_{\nu}+\left[n_{\nu}(m-2)-m_{\nu}\right]}{\left(x_{\nu}-x_{k}\right)^{m_{k}-i-j}} \\
& =\frac{m_{k} ! c_{m_{k}, k, m}}{i ! n_{k}(m-2)}\left\{\left(m_{k}-i\right) b_{m_{k}-i, k, m-2}+\sum_{j=0}^{m_{k}-1-i} b_{j, k, m-2} \sum_{\nu \neq k} \frac{n_{\nu}(m-2)-m_{\nu}}{\left(x_{\nu}-x_{k}\right)^{m_{k}-i-j}}\right\}
\end{aligned}
$$

It remains to compute

$$
S:=\sum_{j=0}^{m_{k}-1-i} b_{j, k, m-2} \sum_{\nu \neq k} \frac{n_{\nu}(m-2)-m_{\nu}}{\left(x_{\nu}-x_{k}\right)^{m_{k}-i-j}} .
$$

It is particularly simple for even $m$; in this case we have $m_{\nu}=n_{\nu}(m-2)$ and hence $S=0$.

Determination of $S$ for odd $m$ in the same way first leads to a formula of different structure:

$$
\begin{aligned}
\sum_{\nu \neq k} \frac{n_{\nu}(m-2)-m_{\nu}}{\left(x_{\nu}-x_{k}\right)^{m_{k}-i-j}} & =\sum_{\nu \in\{0, n+1\} \backslash\{k\}} \frac{1}{2\left(x_{\nu}-x_{k}\right)^{m_{k}-i-j}} \\
& =\frac{1}{\left(m_{k}-i-j-1\right) !} \sum_{\nu \in\{0, n+1\} \backslash\{k\}}\left[\left(2\left(x_{\nu}-x\right)\right)^{-1}\right]_{x=x_{k}}^{\left(m_{k}-i-j-1\right)}
\end{aligned}
$$

Using the notation (1.14) we can get a simple formula for $S$ :

$$
\begin{aligned}
S & =\sum_{j=0}^{m_{k}-1-i} b_{j, k, m-2} \frac{1}{\left(m_{k}-i-j-1\right) !} \sum_{\nu \in\{0, n+1\} \backslash\{k\}}\left[\left(2\left(x_{\nu}-x\right)\right)^{-1}\right]_{x=x_{k}}^{\left(m_{k}-i-j-1\right)} \\
& =\frac{1}{\left(m_{k}-i-1\right) !}\left\{\sum_{\nu \in\{0, n+1\} \backslash\{k\}}\left[2\left(x_{\nu}-x\right) L_{k, m-2}(x)\right]^{-1}\right\}_{x=x_{k}}^{\left(m_{k}-i-1\right)} \\
& =B_{m_{k}-i-1, k, m-2} .
\end{aligned}
$$


By substituting the values of $S$ we obtain a simple formula:

$$
\begin{aligned}
& \sum_{\nu \neq k} c_{m_{\nu}, \nu, m} f^{\left(m_{\nu}\right)}\left(x_{\nu}\right) \\
& \quad=\frac{m_{k} ! c_{m_{k}, k, m}}{i ! n_{k}(m-2)}\left\{\left(m_{k}-i\right) b_{m_{k}-i, k, m-2}+\frac{1}{2}\left[1+(-1)^{m+1}\right] B_{m_{k}-i-1, k, m-2}\right\}
\end{aligned}
$$

At last, from (3.3) we get the recurrence relation (1.17) with respect to the index $m$. Finally, (1.18) follows from (1.16), (1.17), (2.14), (2.15), and the relation

$$
U_{n}^{\prime}\left(x_{k}\right)=\frac{(-1)^{k+1}(n+1)}{1-x_{k}^{2}}
$$

To prove (1.19) by the same arguments as those in $\left[9\right.$, Lemma 3] we claim $b_{i k m}>0$ for even $i$. Thus if both $i$ and $m$ are even then for $1 \leqslant k \leqslant n$ by (1.16) and (1.17)

$$
c_{i k m} \geqslant c_{i, k, m-2} \geqslant c_{i, k, i+2}=\frac{\pi\left(1-x_{k}^{2}\right)^{i / 2}}{(i ! !)^{2}(n+1)^{i+1}} .
$$

\section{REFERENCES}

[1] M. Abramowitz and I.A. Stegun, Handbook of mathematical functions with formulas, graphs and mathematical tables (National Bureau of Standards, Applied Mathematics 55, Washington, D.C., 1965).

[2] S. Bernstein, 'Sur les polynomes orthogonaux relatifs á un segment fini', J. de Math. 9 (1930), 127-177 10 (1931), pp.219-286.

[3] B.D. Bojanov, D. Braess and N. Dyn, 'Generalized Gaussian quadrature formulas', $J$. Approx. Theory 46 (1986), 335-353.

[4] I.S. Gradshteyn and I.M. Ryzhik, Table of integrals, series and products (Academic Press, New York, 1980).

[5] I.P. Natanson, Constructive function theory I-III (Ungar, New York, 1964-1965).

[6] Y.G. Shi, 'A solution of Problem 26 of P. Turán', Sci. China (to appear).

[7] Y.G. Shi, 'On Problem 26 of P. Turán', Acta Sci. Math. (Szeged), (to appear).

[8] Y.G Shi, 'A class of functions having the same best approximation for all $L_{p}$ norms $(1 \leqslant p \leqslant \infty)$ ', Approx. Theory Appl. 5 (1989), 69-72.

[9] J. Szabados, 'On the order of magnitude of fundamental polynomials of Hermite interpolation', Acta Math. Hungar 61 (1993), 357-368.

[10] P. Turán, 'On some open problems of approximation theory', J. Approx. Theory 29 (1980), 23-85.

[11] P. Vértesi, 'Hermite-Fejér interpolations of higher order. I', Acta Math. Hungar. 54 (1989), 135-152. 
Institute of Computational Mathematics and Scientific/Engineering Computing

Chinese Academy of Sciences

PO Box 2719

Beijing

China 100080 